\title{
Neonatal varicella: varicella zoster immunoglobulin (VZIG) does not prevent disease
}

Lucy Reynolds, Siske Struik, Simon Nadel

\begin{abstract}
Two infants with severe varicella are reported. They received varicella zoster immunoglobulin (VZIG) without concurrent information to parents or carers regarding further care. In both these cases there was a three day delay between the onset of symptoms and initiation of aciclovir. This delay was due to lack of awareness of the high risk of varicella in these infants.

Infants born to mothers with onset of chickenpox 4 days before to 2 days after delivery are at risk of fatal varicella, despite the use of VZIG prophylaxis. (Arch Dis Child Fetal Neonatal Ed 1999;81:F69-F70)
\end{abstract}

Keywords: varicella zoster; immunoglobulin; prophylaxis

\section{Case reports}

CASE 1

An Asian infant boy was taken to the local hospital at 11 days of age, by which time he had had a three day history of increasing feeding difficulties and lethargy. On the day of admission he developed diarrhoea and a rash. He had been delivered at term, one day after his mother had developed varicella. The baby had been given $250 \mathrm{mg}$ intramuscular varicella zoster immunoglobulin (VZIG) at 4 hours of age and was breastfed.

Examination showed that the baby had respiratory failure, was shocked, and jaundiced. $\mathrm{He}$ had an extensive erythematous maculo-papular rash with one vesicle present. He required immediate intubation, ventilation, and circulatory support and was given intravenous aciclovir and broad spectrum antibiotics.

He required six days of ventilation, including three days of high frequency oscillatory ventilation (HFOV). Once extubated, he required facial oxygen for a further week.

Immunofluorescence on endotracheal aspirate was strongly positive for varicella zoster virus (VZV), and VZV DNA was demonstrated by polymerase chain reaction of endotracheal aspirate, cerebrospinal and vesicle fluids. There were no positive bacterial cultures. The baby received a total of 16 days of intravenous aciclovir and made a complete recovery. $\mathrm{He}$ remained well 20 months later.

Paediatrics

Paediatric Infectious

Diseases Unit

St Mary's Hospital

Medical School

London W2 1NY

L Reynolds

S Struik

S Nadel

Correspondence to: Dr Simon Nadel.

Accepted 4 March 1999 calamine lotion and paracetamol. He became increasingly unwell and presented to the local accident and emergency department the following day with respiratory distress, irritability, poor feeding and hypoglycaemia. He had an extensive erythematous maculo-papular and vesicular rash. He required fluid resuscitation, intubation, and ventilation. He was ventilated for 14 days, including nine days of HFOV. He required intermittent nasal continuous positive airway pressure (nCPAP) for a further 12 days, and subsequently low flow oxygen via nasal cannulae for six weeks. He developed pancytopenia and clotting derangement and was treated with granulocyte colony stimulating factor, intravenous immunoglobulin, and received several blood transfusions.

The baby also developed seizures. Computed tomography of the brain was normal, but repeated electroencephalography revealed multifocal epileptiform abnormalities. These had improved spontaneously by discharge from hospital after nine weeks.

Varicella zoster antigen was detected on immunofluorescence of material swabbed from a skin vesicle, and from cells obtained by endotracheal aspirate. The virus was subsequently cultured from vesicle fluid. There were no positive bacterial cultures. Intravenous aciclovir was given for 18 days.

This baby remained well at 1 year of age. His development is being closely monitored.

\section{Discussion}

Up to 50 per cent of neonates may become infected with varicella if the mother develops chicken pox four days before delivery and up to two days afterwards. ${ }^{1}$ In these babies the fatality is as high as $31 \%$ without prophylaxis or treatment. ${ }^{2}$ The use of VZIG can modify the clinical course, usually preventing more severe infection. ${ }^{3}$ However, although decreased, the risk of death is not eliminated. ${ }^{45}$ Therefore, VZIG does not always prevent severe or fatal varicella in this high risk group. Expectant treatment with close observation, followed by prompt initiation of antiviral treatment on suspicion of neonatal varicella, is recommended.

In both these cases there was a three day delay between the onset of symptoms and initiation of aciclovir. This delay was due to lack of awareness of the high risk of varicella in these infants. The average incubation period from the onset of the mother's rash to onset of the baby's rash is 11 days (range one to 16 days). ${ }^{1}$ As administration of VZIG can prolong the incubation period, this period of vigilance should extend to 30 days. VZIG may also modify disease and may lead to atypical rashes, such as occurred in the infants reported here. 
When a neonate who has received VZIG is discharged home, it should be made clear to the parents and all health workers involved that if the baby becomes unwell in any way and/or develops any sort of rash, prompt hospital review, with the possibility of intravenous aciclovir, should be undertaken.

\section{Conclusions}

Despite appropriate intervention with VZIG, the possibility of varicella infection was not appreciated by either the parents of these infants or the health professionals involved in the babies' care. Therefore, appropriate treatment was delayed, with potentially fatal consequences. We advocate an information leaflet for the families of such babies (preferably in the family's first language as well as English). Clear instructions to parents and health professionals may prevent avoidable morbidity and mortality in this group of patients.

1 Gershon AA. Chickenpox, measles and mumps. In: Remington JS, Klein JO, eds. Infectious diseases of the fetus and newborn infant. 4th Edn. Philadelphia: WB Saunders, 1995:565-618.

2 Meyers JD. Congenital varicella in term infants: Risk reconsidered. F Infect Dis 1974;129:215-17.

3 Hanngren K, Grandien M, Granstrom G. Effect of zoster immunoglobulin for varicella prophylaxis in the newborn. Scand F Infect Dis 1985;17:343-7.

4 Holland P, Isaacs D, Moxon ER. Fatal neonatal varicella infection. Lancet 1986;ii: 1156.

5 King S, Gorensek M, Ford-Jones EL, Read SE. Fatal varicella-zoster infection in a newborn treated with varicella-zoster immunoglobulin. Pediatr Infect Dis 1986;5:558-9. 